\title{
Conciencia plena en Kent Wilber y Karl Jaspers
}

\section{Full conscience in Kent Wilber and Karl Jaspers}

Margarita Morandé-Dattwyler (margarita.morande@uach.cl) Facultad de Medicina, Universidad Austral de Chile (Valdivia, Chile) ORCID: 0000-0001-7349-2884

\begin{abstract}
The following article exposes the theories of Karl Jaspers and Kent Wilber regarding the possibility of a consciousness that accesses the totality of being. Although the question of being responds to a philosophical search, both authors are referents in the field of mental health. For them, some reference texts were taken, from which it was possible to identify the different stages established by the authors towards full consciousness, as well as some similarities and theoretical differences that they could present. In this way, the question of participation in being, such as states of consciousness, allows a greater understanding of the human being and a fruitful exchange between philosophy and the different disciplines of mental health.
\end{abstract}

Key words: conscience, being, Jaspers, Wilber, stages, mental health.

\section{Resumen}

El siguiente artículo expone las teorías de Karl Jaspers y Kent Wilber respecto a la posibilidad de una conciencia que accede a la totalidad del ser. Si bien la pregunta por el ser responde a una búsqueda filosófica, ambos autores son referentes en el campo de la salud mental. Para ello, se tomaron algunos textos de referencia, desde los cuales fue posible identificar las distintas etapas establecidas por los autores hacia la consciencia plena, además de algunas semejanzas y diferencias teóricas que pudiesen presentar. De esta manera, la pregunta por la participación en el ser, como los estados de consciencia, permiten una mayor comprensión del ser humano y un intercambio fructífero entre la filosofía y las distintas disciplinas de la salud mental.

Palabras clave: conciencia, ser, Jaspers, Wilber, etapas, salud mental.

\section{Introducción}

En el devenir de la historia filosófica, es posible constatar que la pregunta por el ser ha sido una pregunta que ha interpelado a los seres humanos, en cuanto seres que existen y que son capaces de preguntarse por su existencia. Sin duda, dicha pregunta ha tenido distintas aristas, muchas de las cuales se relacionan con el origen del ser, con su manifestación y la participación en éste.

Tanto para Jaspers como para Wilber, la participación en el ser, en cuanto ser total, se relacionan con nuestros estados de conciencia. Así, en Jaspers encontramos el concepto de conciencia absoluta, mientras que en Wilber el concepto de conciencia de unidad. Si bien, ambos autores presentan diferencias en la 
forma de abordar la problemática y en la forma de acceder a dicho estado de conciencia, también es cierto que existen ciertas similitudes. Por eso, es que se ha querido establecer qué se entiende por conciencia plena para ambos autores (como una forma de participar en plenitud del ser, que es único e indivisible), tomando como referencia dos textos. El primero, es el texto de Jaspers Philosophie. El segundo texto es de Wilber La conciencia sin fronteras. Aproximaciones de Oriente y Occidente al crecimiento personal, donde el autor expone su teoría acerca del espectro de la conciencia.

Uno de los aspectos interesantes de abordar en ambos autores, es que los dos son referentes teóricos para la salud mental, Jaspers en cuanto a su método fenomenológico en la psiquiatría y Wilber como uno de los exponentes de la corriente de la psicología transpersonal. A continuación, se exponen brevemente sus respectivos planteamientos teóricos, para luego dar comienzo a su análisis.

\section{Conciencia absoluta en Jaspers}

Para Jaspers, ante todo, los seres humanos somos ser-ahí (no más), ya que existimos en el mundo y estamos determinados por nuestras necesidades y la satisfacción de poder. Este ser ahí no más está caracterizado, entre otros, por la costumbre, la opinión general y las necesidades de subsistencia, todo lo cual evidencia su incapacidad para el destino. De esta manera, su conciencia temporal se limita a los apremios cotidianos, viéndose movido e impulsado por distintas situaciones que lo sitúan junto a sus congéneres, sin el camino de una vida.

Es así como el ser humano va objetivizando distintos aspectos de su vida, identificándose con ellos. Por ejemplo, puede llegar a creer que su yo se objetiviza en su ser social. Y esto, porque puede identificarse con lo que es para los demás. Así, el autor explica que las acciones que realizo sobre los demás, arrojan una imagen de mi ser, y esta imagen que se vuelve a cernir sobre mí mismo, es lo que puedo llegar a considerar como mi ser. Un ejemplo de esto sería creer que soy lo que determina mi profesión, es decir, mi ser descansa en un rol profesional. Sin embargo, el autor nos advierte sobre esta confusión y nos aclara que, los seres humanos, aunque estemos determinados por nuestro ser-ahí sociológico, permanecemos siendo desde el origen, la posibilidad de mí mismo. Por ello, nuestro yo, no es el resultado de nuestras constelaciones sociales.

Con ello, Jaspers nos advierte que el hombre no agota su ser en lo mundanal, o en las situaciones que se ven caracterizadas por distintas condiciones (físicas, sociológicas, históricas, políticas, psicológicas, etc.), sino que también se ve enfrentado a situaciones límites, que le recuerdan su finitud y que lo obligan a ser desde el origen. De esta manera, aparece la posibilidad de la trascendencia, como posibilidad de trascender lo mundanal.

Esta trascendencia, que correspondería al ser propiamente tal, sitúa al hombre como un ser abierto a la posibilidad y a la libertad. Y esto, porque la trascendencia nunca puede objetivarse, caracterizarse 0 calcularse. Ella se manifiesta como una interpelación o un llamado, haciendo que los hombres la anhelen, la busquen e invoquen. De esta manera, si bien, el ser-ahí solo es posible realizarlo en la cotidianidad de nuestro mundo, de nuestra existencia, la lucha por ser-sí-mismo, se constituye en un acto de trascender por medio de nuestra libertad y posibilidad. Así, la definición del yo-mismo, no se acaba en mi ser yocuerpo, yo-carácter, yo-rol, o todas aquellas condiciones que caracterizan nuestro ser-ahí, sino antes bien, se afirma como posibilidad y libertad en el acto de trascender. Por ello, el hombre se encuentra en constante tensión entre lo mundanal y lo trascendente, entre el ser-ahí y la existencia. Como señala el 
autor, "la conciencia absoluta no es 'forma de vida', 'ni actitud', ni 'posición intelectual'” (Jaspers 1959:135).

Un aspecto importante a considerar al respecto, es que la libertad se muestra a través de la acción. De esta manera, se asumiría el origen de lo que soy, como fundamento a todas las acciones determinadas, a partir del cual he querido y tenido que querer.

Ahora bien, así como la libertad vendría a ser una determinación esencial de nuestro ser, también lo sería la posibilidad de asumirnos como seres posibles. Así, el ser humano, desde su libertad y posibilidad, tiene el desafío de edificarse a sí mismo. Por ello, la posibilidad de ser-sí-mismo, solo puede ser alcanzada desde la libertad de trascender los esquemas mundanales, de trascender mi ser-ahí-no más, lo cual supone un salto y un compromiso por entero de aquellas decisiones que tomo. De esta manera, la existencia o manifestación del ser, interpela, llama y se manifiesta. Y esto, sobre todo en aquellas ideas del espíritu que son capaces de transformar la cotidianidad. En la resolución, se experimenta la libertad de elegirse uno mismo, así cada yo mismo, es la libertad de esa elección.

Jaspers llamaría "resolución" a aquella elección primaria y anterior por uno mismo (vínculo claro y definitivo conmigo mismo), que nos permite asumirnos como individuos libres y no dejarnos arrastrar por lo que otros hacen y opinan ante la duda de qué hacer. En el caso del autor, esta opción primaria es tan radical, que no estaría sujeta al éxito o fracaso, siendo esta resolución verdadera, aun cuando se fracase en la posibilidad de ser-sí-mismo. De una manera muy particular el autor señalaría que no se puede abandonar dicha elección, porque si lo abandono, me destruyo a mí mismo. Es por eso que se sostendría incondicionalmente. Voy tras ello, como yo mismo. De esta manera, la conciencia absoluta, "como manifestación de la 'existencia' es la presencia de lo incondicionado que soy yo mismo, el ser, responsable del cual yo enfrento conmigo como mi propio ser. Es el cumplimiento positivo por virtud de la experiencia de la acción interior" (Jaspers 1959:134). Así, "lo que es la conciencia absoluta lo experimento activamente cuando estoy cierto de mí como libertad cumplida" (Jaspers 1959:134).

Ahora bien, para Jaspers, la conciencia es la que nos orienta en el mundo. Sin embargo, él logra distinguir tres conciencias distintas. Una es la conciencia de la vivencia que se hace presente. Por ejemplo, somos conscientes de lo que comemos, qué nos ponemos, con quién estamos, etc. Otra, es la conciencia que caracteriza a la condición universal de estar consciente, y que permite identificar y canalizar la relación sujeto-objeto. Y un tercer tipo de conciencia es la conciencia absoluta. Esta conciencia va más allá de la relación sujeto-objeto; es la certidumbre-del-ser, de lo envolvente, ella "penetra y transforma toda la existencia empírica vivida" (Jaspers 1959:133). A través de ella, el ser mismo se nos presenta en lo inmediato, porque ella se abre al fundamento del ser, como una actitud existencial. También es importante señalar que ella no se encuentra encapsulada o acotada a una forma de vida, regla del comportarse, postura frente a la relación sujeto-objeto o una actitud espiritual que permite afectarnos por ideas. Ella es dinámica y se constituye en el motor que mueve desde el origen: "Existe como libertad, a lo que no le corresponde otro ser que el ser en su propia acción" (Jaspers 1959:136). Y ese movimiento, tiende al camino de la plenitud, de la consumación, la cual se expresaría en tres condiciones de realización: amor, fe y fantasía.

Si se realiza el recorrido desde el movimiento del origen, es posible reconocer un primer momento en que hay un reconocimiento de los propios límites. En este momento, se lleva a cabo una operación filosófica fundamental donde se reconoce que no es posible objetivizar o esquematizar la existencia, teniendo como resultado un no-saber o el saber del no-saber. Este momento de no saber, permite hacer el tránsito 
necesario en el camino del querer saber, el cual no tiene ningún límite. Así Jaspers aclara que el movimiento de la conciencia absoluta, no es como un saber general de mi no-saber, "sino como un nosaber conquistado, que en cada caso se sabe a sí mismo al anularse un saber objetivo" (Jaspers 1959:139).

Luego, nos encontramos ante el vértigo de reconocer que cualquier respuesta nos confronta con una contradicción. Y es que una afirmación, como el sentido o el fundamento último, puede ser justificada de igual manera en su negación. Por ejemplo, si afirmamos la existencia de un fundamento último, estamos diciendo al mismo tiempo que este fundamento no tuvo ningún fundamento, generando un abismo entre la afirmación y la negación, entre el ser y la nada. Así, frente a este escenario, o encontramos una salida al ser pleno, o nos sumergimos en la nada absoluta, entre lo pensable y lo impensable. $Y$ esto, exige abandonar toda intención de representar u objetivar de alguna forma, iniciando el camino hacia una conciencia absoluta. El vértigo, "impulsa abandonando la ilusión engañosa de lo superficial, hacia el verdadero ser o hacia la verdadera nada; entonces se encuentra de golpe ante la decisión" (Jaspers 1959: 145).

Pero la posibilidad de la nada enfrenta a los hombres con la angustia existencial, la angustia de perdernos a nosotros mismos, y con ello, a la culpa de estar consciente del vacío de mi propio ser. Y es que la culpa está entrañablemente ligada a la posibilidad de elección. Como señala claramente el autor, "la angustia es el vértigo y el espanto que experimenta la libertad al encontrarse forzada a elegir. Solamente superando la angustia se puede alcanzar la decisión y firmeza de la conciencia absoluta" (Jaspers 1959:143). Así, sin la certeza de saber qué elegir, el hombre se entrega pasivamente al arbitrio de los acaecimientos. Y es que la angustia existencial no puede ser superada por medio de ninguna medida técnica o concreta como en el ser-ahí, es necesario sumergirse en ella y atravesarla sin ninguna garantía objetiva.

De esta manera, se hace necesario trascender lo mundanal para que, por medio de la conciencia, hable el ser propio de cada uno. Es en la conciencia donde habla el yo mismo o el sí-mismo, que ha tomado distancia del ser-ahí. Y esta distancia con el ser-ahí, es la que permite que se manifieste el ser-posible. Asimismo, la conciencia como receptora de nuestro sí-mismo, sería la que recibiría el mandato incondicional que, en relación con el mundo, otorga una dirección determinada. De tal modo que lo que queramos hacer, lo que se revela como sí-mismo en el hacer, lo queramos hacer eternamente, dando siempre una respuesta afirmativa al ser. Así "lo que yo hago debe ser de tal suerte que yo pueda querer que el mundo en general sea de tal modo que ello tenga que acontecer dondequiera" (Jaspers 1959:148).

Es así como la conciencia lleva al hombre a la acción que se desprende de una resolución existencial, que es independiente de las conveniencias, como la resolución finita; ella más bien es una resolución de elegirnos a nosotros mismos, como posibilidad de ser sí-mismos. Y esta posibilidad es la que nos "completaría" como un ser entero, un ser que es resoluto ante la conciencia absoluta. Por eso, si bien, la conciencia puede fortalecerse en el ser-ahí temporal, está caracterizada por la intimidad e individualidad que, siendo inaccesible para otros y nosotros mismos, se realiza en la comunicación con otros. Es el origen moviente de la verdad de mí ser.

Ahora bien, el resultado de este camino para Jaspers sería la posibilidad de hacerse plenos (plenitud dinámica), de completarse o consumarse, lo que se realizaría en este mundo, por medio de las experiencias del amor, la fe y la fantasía. De esta manera, Jaspers señala que "la conciencia absoluta puede esclarecerse como amor, que es creencia activa y conduce a la acción incondicionada, y se convierte en fantasía contemplativa y en conjuro metafísico. Todo lo que de ella emana está en indisoluble correlación" (Jaspers 1959:155). 
Así, la plena realización de la existencia se daría en la unión con lo Otro (humano u otro ser), orientándose a la realización del instante pleno y desde el cual, toda orientación temporal cobra verdadero sentido. A su vez, este amor se da en un perpetuo movimiento de llegar al ser, es un amor que no se apoya en razones, que no tiene fundamento, pero que, a partir de él, todo tendría su fundamento y razón de ser en el amor. "El que ama no está sobre lo sensible en un más allá, sino que su amor es la indiscutida presencia de la trascendencia en la inmanencia, lo maravilloso aquí y ahora" (Jaspers 1959:156). Asimismo, "el amor es infinito; no sabe de un modo objetivo qué y por qué ama, ni puede encontrar en sí mismo un motivo. Por el amor queda fundado lo que es esencial" (Jaspers 1959:156).

También es un amor infinito que se manifiesta en la espontaneidad como entrega-de-sí y que, por este medio, llegamos a ser sí-mismos. Es siempre un salir de sí-mismo para fundirse parcialmente con el Otro, lo que termina transformándonos y transformando como personal, todo ser-ahí. "El amor tiene su profundidad en la relación de 'existencia' a 'existencia'. Entonces, para el amor, toda existencia empírica se hace como si fuera personal” (Jaspers 1959:157). A su vez, el amor es lealtad. En él se encuentra la absoluta confianza, sin cálculos de ningún tipo. Finalmente, Jaspers señala que sin amor todo se hundiría en la nada.

Por su parte, la fe, en cuanto fe filosófica, es la certidumbre-del-ser. Es una certidumbre que no se puede poseer (tomar posesión), pero que tampoco se reduce a una mera opinión. Es una interpelación. Así, por una parte, es el modo en que el alma es cierta de su ser, de su finalidad, y por otra, una expresión de contenidos que luego desaparecen, ya que no se pueden absolutizar. Es fe en este mundo, en cuanto la existencia se revela en él. A diferencia del saber, que toca lo finito del mundo y lo hace posesivo, la fe toca el ser propio. Es confianza en el ser, y con ello, esperanza, ya que es capaz de llegar hasta donde el saber no llega: la posibilidad de existencia y trascendencia. Y esto, nos determina existencialmente, siendo la única capaz de sostener al hombre cuando se ha perdido todo.

Finalmente, la fantasía, que al igual que en la fe está presente el amor, nos lleva a descubrir la presencia del ser en el ser-ahí (en la inminencia), transportándolo a otra dimensión. Y la manera en que opera es por medio de lo que queda como una cifra, lo que no se agota en el puro saber o lo que ha sido objetivado. Así, la fantasía opera de manera intuitiva o imaginativa, o bien, como pensante y especulativa: "Me permite experimentar lo perfecto, lo que descansa en sí mismo" (Jaspers 1959:162). Es certidumbre-delser, pero que se revela en un lenguaje simbólico, permitiendo que el ser nos transforme.

En último término, Jaspers termina el recorrido señalando cómo es posible mantener o resguardar la experiencia de una conciencia absoluta en nuestra mundalidad, para lo cual establece cuatro actitudes existenciales que ayudarían a este fin: la ironía, el juego, el pudor y la serenidad. Lo común a todas ellas, es que, de cierta forma, permiten mantener distancia con el ser-ahí, sin caer en la objetivación del ser, pero favoreciendo que se mantenga como algo cierto.

\section{Conciencia de unidad en Wilber}

Para Wilber, la unidad de consciencia o identidad suprema constituye la naturaleza y condición de todos los seres sensibles, sin embargo, paulatinamente limitamos nuestro mundo estableciendo fronteras que lo dividen en diversos niveles, haciendo que nuestra conciencia opere en estos niveles. Así, cuando las personas responden a la pregunta "¿quién soy?", lo que en realidad hacen, es trazar un límite mental que atraviesa el campo de la experiencia. De esta manera, todo lo que queda dentro de ese límite, se percibe como "yo mismo" y todo lo que queda fuera, es algo excluido del "yo mismo". Así, cada nivel del espectro 
de conciencia, representa un estrechamiento o restricción de lo que el individuo siente que es él mismo, su verdadera identidad: "Tan fundamental es la demarcación primaria entre el yo que somos y lo ajeno a ese yo, que de ella dependen todas las otras demarcaciones que establecemos. Mal podríamos distinguir límites entre las cosas mientras no nos hayamos distinguido de las cosas" (Wilber 1984:69).

Una vez trazadas las líneas generales, las respuestas pueden ser sumamente complejas o simples, pero seguirán siendo dependientes del trazado inicial. A su vez, en el acto de ir trazando demarcaciones (tomar decisiones, desear, aprender, etc.), se van creando mundos de opuestos, que no existían por sí mismos, creando con ello un mundo de potenciales conflictos. Por ejemplo, cuanto más alguien se aferra al placer, tanto más teme el dolor. Generalmente, esta dualidad se resuelve extirpando uno de los opuestos o reduciéndolo al otro, pero nunca se cuestiona la demarcación como tal. Sin embargo, si bien ambos opuestos son muy distintos, están unidos en su existencia, y la dificultad está en la tendencia de considerarlos irreconciliables. Esta es la distinción entre una línea y una frontera: "Lo importante es, pues, que las líneas unen los opuestos en la misma medida que los distinguen, y tal es, por cierto, la esencia y la función de todas las líneas y superficies reales en la naturaleza. Delimitan explícitamente los opuestos, al mismo tiempo que implícitamente los unifican" (Wilber 1984:45).

La totalidad de las líneas que encontramos en la naturaleza, e incluso las que trazamos nosotros mismos, no distinguen simplemente los diferentes opuestos, sino que también los une en una unidad inseparable: “En otras palabras, una línea no es una demarcación, porque una línea, ya sea mental, natural o lógica, no solo divide y separa, sino que también une y aproxima. Por otra parte, las fronteras son puras ilusiones: fingen separar lo que de hecho no es separable. En este sentido, el mundo real contiene líneas, pero no tiene fronteras" (Wilber 1984:45).

Tal como señala el autor, "el secreto metafísico fundamental, si nos atrevemos a enunciarlo de manera tan simple, es que no hay fronteras en el universo. Las fronteras son ilusiones, demarcaciones; no son productos de la realidad, sino de la forma en que la cartografiamos y la acotamos. Y aunque esté muy bien cartografiar el territorio, confundir el territorio y el mapa es fatal" (Wilber 1984:51).

De acuerdo al autor, existirían muchas formas de demarcación en la historia. Entre ellas están las palabras que producen una clase, la clase de una clase o números, la meta-meta demarcación o variables, que establecen leyes y principios, etc. Esto no quiere decir que el mundo real sea solo producto de nuestra imaginación, sino que las demarcaciones lo son. Así, cuando se dejan de lado las apariencias y toda discriminación cesa, queda la naturaleza esencial y verdadera de las cosas. "El mundo contiene toda clase de rasgos, superficies y líneas, pero están todas entretejidas en una trama sin costura" (Wilber 1984:66). Revelar que la realidad no tiene fronteras es señalar que los conflictos son ilusorios; la batalla no se resuelve, se disuelve.

En el campo de la conciencia, "la conciencia de unidad no es más que la aprehensión del verdadero territorio sin demarcaciones" (Wilber 1984:68). La demarcación de lo que somos y no somos, es la primera en trazarse y la última en borrarse. Es la escisión entre sujeto cognoscente y objeto conocido. En la conciencia de unidad, el yo se expande hasta incluir todo lo que se creyó ajeno. Una vez comprendido el carácter ilusorio de la demarcación primaria, el sentimiento de la propia identidad alcanza el Todo. La demarcación primaria parecía separar al hombre de la experiencia, pero ella se desvanece en la experiencia. Así, la conciencia de unidad es el tiempo presente, sin fin, simple y accesible: "Los sabios iluminados de todos los tiempos y de todas las latitudes coinciden en que la consciencia de unidad no es temporal, no se da en el tiempo, sino que es intemporal y eterna. No sabe de comienzo y nacimiento, ni 
de acabamiento y muerte" (Wilber 1984:87). En este contexto, los conflictos nacen de la preocupación por el pasado y futuro. Aquí, no se trata de ignorarlos o negarlos, sino de reconocerlos como demarcaciones. $Y$ esto porque la experiencia de un pasado o un futuro solo se da en el presente. De esta manera, en la conciencia de unidad, no se huye del tiempo, se abraza su totalidad. Se puede reflexionar libremente sobre el pasado y el futuro sin estar sujeto a ellos.

El autor agrega que, si la realidad es efectivamente una condición en que no hay demarcaciones, entonces la consciencia de unidad es el estado natural de la consciencia que reconoce esta realidad: es la comprensión de lo que no tiene demarcaciones. Así, si bien se podría llegar a concluir que para llegar a la conciencia de unidad hay que destruir la demarcación primaria, ni siquiera habría que tomarse esa molestia, porque ésta no existe. Como todas las fronteras, no es más que una ilusión.

Señala que los hombres se han ido identificado con su cuerpo, su mente, y su personalidad, imaginando que ellos constituyen su verdadero ser, y de esta manera, se pasan la vida procurando defender, proteger y prolongar lo que no es más que una ilusión, mientras la identidad suprema aguarda que la descubramos. En este proceso, habría que reconocer la dificultad de describir la experiencia, porque nuestras palabras e ideas son demarcaciones, mientras que el ser verdadero es una percepción de lo que carece de fronteras. Por ello, hay que contentarse con señalar y mostrar un camino por el cual se pueda tener la experiencia de conciencia de unidad.

Agrega que el mundo de lo real no es una colección de cosas separadas e independientes. Cada cosa y acontecimiento en el cosmos, se interrelaciona con y depende de cada una de las otras cosas y acontecimientos. Son nuestros mapas simbólicos y nuestras demarcaciones lo que nos da ilusión de entidades independientes. Así, el descubrimiento del mundo real, de lo que carece de fronteras, es consciencia de unidad. No se trata de que en la consciencia de unidad esté mirando el territorio real sin demarcaciones, sino más bien, que la consciencia de unidad es ese territorio. La realidad es percepción de lo que no tiene fronteras, lo mismo que nuestro verdadero ser.

En relación al espectro de la consciencia, el autor señala que se puede entender cada nivel como una demarcación progresiva que limita el verdadero ser de la conciencia de unidad. A continuación, se describe cada nivel, partiendo por ésta última.

En el nivel de la conciencia de unidad, ya no hay demarcaciones o estados parciales, ya que es la condición y naturaleza de todos los estados. Es la experiencia presente tal como es, está siempre y en todas partes. Aquí el fin y los medios, el trayecto y el destino, son una y la misma cosa. Alcanzarla significa que ya se expresa como la verdadera naturaleza. Para esto, hay que disolver la resistencia primaria con el fin de mirar el presente global. Al resistir la experiencia presente, el hombre se separa de ella reduciéndose al presente fugitivo. Así, mientras el hombre no vea que todo lo que hace es apartarse, pensará que tiene que hacer algo, realizando algún movimiento que nuevamente lo aparta. Pero en el punto donde ve que todo lo que hace es resistencia, no le queda más opción que entregarse, porque la unidad ya es en todo momento. Y cuando ya no hay resistencia a la experiencia presente, no hay motivo para separarse de ella: "Como la conciencia de unidad es conciencia del momento intemporal, está totalmente presente en el ahora, y, como es obvio, no hay manera de alcanzar el ahora, de llegar a lo que ya es" (Wilber 1984:185).

En el nivel de las bandas transpersonales, se establece una demarcación entre lo individual-personal y lo supraindividual-trascendental. Ella trasciende las demarcaciones de espacio y tiempo, y mira al individuo con los ojos del espíritu colectivo de la humanidad. Así, el individuo deja de identificarse exclusivamente 
con su cuerpo o mente, sin sofocarse en dramas personales. Su ser más profundo puede trascenderlos manteniéndose intacto, libre y abierto como testigo transpersonal. Por medio de un proceso de desidentificación, se pueden observar las aflicciones personales sin juzgarlas, justificarlas, evitarlas, manipularlas o dramatizarlas, porque se entiende que ninguna de ellas constituye el verdadero ser. Aquí es posible relacionarse con el cuerpo y la mente, como con todos los demás objetos. Así se establece contacto con todos los niveles de conciencia, sin estar limitado por ellos. Asimismo, la intuición de trascendencia lleva aparejada la intuición de inmortalidad. El hecho, que no haya ningún momento que no se experimente "uno mismo", significa que algo de "uno mismo" queda intacto pese al tiempo, como las preocupaciones, experiencias, pensamientos, emociones, entorno y sensaciones experimentadas. Se experimenta la misma yoidad.

El nivel de Centauro (unidad psicosomática), se inicia en la demarcación primaria, aquella que divide el organismo frente al medio. El hombre se identifica solo con su organismo y se opone al medio, y esto hace que comience a preocuparle la muerte. Quiere algo que circunde su presente para protegerse de la muerte, poniendo como fronteras el pasado y futuro. Sin embargo, en este nivel aún está en contacto con su ser psicofísico, por eso se le llama centauro. En este nivel, se establece una demarcación entre el cuerpo y el ego, siendo lo más significativo la asociación del cuerpo con la muerte y la pugna entre lo voluntario y lo involuntario. Así, el ego suele identificarse con los procesos voluntarios y considera lo incontrolable como indigno de confianza, se resiste a la atención sensible del centauro.

En el nivel siguiente, nivel del ego, el hombre se niega a permanecer en contacto con la totalidad de su organismo y se identifica con su ego, su autoimagen, su personalidad, puramente mental. Rechaza el cuerpo convirtiéndolo en propiedad. Esto, porque el hombre sabe que su cuerpo es mortal y en busca de la inmortalidad estática, se centra en una idea de sí mismo. "La palabra 'árbol', por ejemplo, sigue siendo la misma por más que todos los árboles reales cambien, crezcan, se transformen o se sequen. En busca de esta inmortalidad estática, el hombre empieza, por consiguiente, a centrar su identidad en torno a una idea de sí mismo; y ésa es la abstracción mental que llamamos el 'ego"' (Wilber 1984:111). Así la línea natural entre cuerpo y mente se transforma en una frontera. El hombre no puede encontrar una imagen exacta y aceptable de sí mismo porque la ha desfigurado. Se genera el nivel de la persona.

En el nivel de la persona se establece una demarcación entre lo que nos gusta de nosotros mismos (persona) y lo que no nos gusta (sombra). Estas facetas negadas, son proyectadas haciéndolas parecer como parte del medio o de otros, propiciando un campo de batalla con la sombra. Así, elaborar una imagen personal más precisa, descendiendo de la persona al ego, consiste en derribar una barrera, reconociendo e incorporando las facetas desconocidas; abriéndose a la comprensión y aceptación de todas las potencialidades, tanto negativas como positivas.

Finalmente, el autor señala que el sufrimiento es el primer paso en el reconocimiento de las falsas demarcaciones y puede ser liberador si es que el hombre no se aferra a él. Hay que interpretarlo, para vivirlo y trascenderlo. Hay que entender qué significa y qué lo produce según el estado particular de conciencia: "No ganaremos absolutamente nada saltando de una ola en otra. Más aún, corremos el riesgo de perder mucho, ya que mientras sigamos saltando olas en busca de la acuosidad, evidentemente nunca descubriremos que la acuosidad se encuentra, en toda su pureza, en la ola sobre la que montamos en este momento. Buscar la conciencia de unidad es como saltar de una ola de experiencia a otra, en busca del agua" (Wilber 1984:187). 


\section{Semejanzas y diferencias en ambos enfoques}

Habiendo expuesto ya ambas teorías, es posible reconocer que ellas presentan algunas diferencias y semejanzas las cuales conviene revisar. En primer lugar, ambas teorías postulan un estado de conciencia en el cual es posible participar del ser y la existencia en toda su plenitud, entendiendo que no es ajena a la vida de los hombres, en la medida que se dejen interpelar por ella, ya que esta experiencia no se puede objetivizar o codificar. Ella es el tiempo y la vivencia presente en toda su magnitud, donde el yo puede ser "yo mismo" o ser-si-mismo. En el caso de Jaspers, además se señala que ella es una condición dinámica, un motor que nos pone en movimiento desde el origen.

Si bien en Jaspers encontramos un trazado para alcanzar esta conciencia, desde el no saber hasta atreverse a atravesar la angustia existencial, y en Wilber encontramos el camino de reconocer la ilusión de las demarcaciones, en ambos es posible constatar que esta experiencia o el ser, no se puede encapsular en aspectos concretos, manipulativos, determinados. Tal como señala Keeney, todos establecemos distinciones para distinguir una pauta de otra, para conocer nuestro mundo, siendo el punto de partida de cualquier acción, percepción, pensamiento, decisión, descripción, etc.: "El punto de partida de la epistemología es, entonces, el observador que establece distinciones a fin de observar; y lo que el observador observa puede ser descripto (...). Las descripciones mismas consisten en establecer distinciones en lo que observamos. Se inaugura así una recursión: establecemos distinciones a fin de observar, y luego establecemos distinciones a fin de describir lo que observamos" (Keeney 1987:39). Pero como señala Wilber, lo que entendemos por "el que ve" no es otra cosa que la experiencia total de lo visto (1984). Así, por ejemplo, si miro una manzana, no hay una experiencia de la manzana y otra que sea ver la manzana, solo hay una experiencia única de ver una manzana. Por ello, resulta coherente abandonar las demarcaciones y el saber, para atravesar y acceder a la conciencia de unidad o conciencia absoluta. Y esto porque la conciencia absoluta no puede ser cosificada.

Otro aspecto común en ambos autores es la trascendencia del dolor en el camino hacia tal estado de conciencia. En el caso de Wilber, el sufrimiento puede ser el primer paso para identificar las falsas demarcaciones, mientras que, en Jaspers, el ser humano se ve enfrentado a situaciones límites, que le recuerdan su finitud y que lo obligan a ser desde el origen. Ahora bien, cabe hacer una distinción entre ambos autores, ya que, en el caso de Jaspers, la muerte obliga al hombre a ser desde el origen, mientras que, en Wilber, ayudaría a separar el organismo del ego, en cuanto éste rechaza la mortalidad de su cuerpo. De esta manera, si bien puede ser un camino hacia la conciencia absoluta o de unidad, dependerá de la forma en que la asumamos y nos sumerjamos en dichas experiencias, permitiendo que ellas sean trascendidas. De lo contrario, desde Wilber, estaremos en permanente conflicto, el cual resultaría de la demarcación entre mi organismo y el medio. Pero recodemos que las demarcaciones son ilusorias.

Otro aspecto a considerar entre ambas teorías es la relación que se podría establecer entre las condiciones del ser-ahí en Jaspers, que hacen que los hombres se limiten a los apremios cotidianos, viéndose movidos e impulsados por distintas situaciones que los sitúan junto a sus congéneres, y los distintos niveles de conciencia en Wilber. Si bien no existe una correlación directa entre ambos, es posible dilucidar que tanto los distintos niveles de conciencia en Wilber, como los condicionantes mundanales en Jaspers, limitan el reconocimiento del ser-si-mismo o el yo mismo. Esto, porque lo asocian a ciertos aspectos limitados de la identidad del yo, como son el yo-organismo, yo-rol, yo-ego, yo-carácter, etc. Sin embargo, a diferencia de Jaspers, Wilber establece un espectro de conciencia que va restringiendo cada vez más la identidad del yo de manera progresiva (conciencia de unidad, bandas trascendentales, centauro, ego y persona), mientras que Jaspers, establece niveles de conciencia que responden a estados cualitativamente distintos 
(conciencia de la vivencia que se hace presente, conciencia universal que establece la relación sujetoobjeto y conciencia absoluta). Esta distinción es la razón por la cual ambos autores no establezcan igual cantidad de conciencias en sus respectivas teorías.

Finalmente, una gran diferencia entre ambos es que Jaspers define un camino de regreso al ser-ahí desde la conciencia absoluta, la cual se caracteriza por sus manifestaciones (amor, fe y fantasía) y que debe ser resguardada de la mundanidad mediante ciertas actitudes existenciales, como son la ironía, el juego, la serenidad y el pudor. Wilber en cambio solo advierte la condición de considerar las demarcaciones como ilusiones y reconocer que una línea no es lo mismo que una demarcación ya que, en esta última, se podría presentar un conflicto que no es constitutivo del ser. Como señalaría Wilber, ni siquiera se trataría de resolver un conflicto, sino de disolverlo, ya que las demarcaciones son meras ilusiones. A su vez, otra de las diferencias entre los autores, es que Jaspers pone énfasis en los conceptos de libertad, posibilidad y resolución existencial, los que no se encuentran en Wilber. Aquí el yo se identificaría como una acción de posibilidad y libertad, empuñada y asumida, en cuanto nos afirmamos como seres posibles y libres, más allá de las esquematizaciones y decisiones. Wilber, por su parte, incorpora los aspectos inconscientes, como la sombra, lo que no es abordado por Jaspers. De esta manera, el inconsciente vendría a ser otra demarcación del yo, que nos apartaría de la conciencia de unidad, al separar lo que nos gusta y no nos gusta de nosotros mismos.

\section{Conclusiones}

El siguiente artículo buscó exponer dos teorías distintas que afirmaban la existencia de una conciencia capaz de acceder al ser (conciencia absoluta y conciencia de unidad), la cual fue conceptualizada por la autora como conciencia plena, en cuanto en ella, es posible acceder a la manifestación del ser en toda su unicidad, magnitud y como espacio de realización del sí mismo o del yo.

Si bien ambas teorías presentan diferencias respecto al detalle de su desarrollo, los niveles que trabajan y algunas significaciones que pueden ser distintas (por ejemplo, la experiencia de la muerte), ambas comparten también ciertas semejanzas que son interesantes de tener presente. Asimismo, no resulta menor que sean dos autores que comparten el interés por la salud mental, llevando sus planteamientos no solo al ámbito filosófico, sino además al campo de la salud de las personas. Esto queda aún más evidente en los planteamientos de Wilber, quien es capaz de ordenar los distintos enfoques psicoterapéuticos de acuerdo al tipo de sufrimiento y estado de conciencia de las personas.

Hoy en día saber que es posible un camino hacia la consciencia plena, nos hace más libres como seres humanos. Asimismo, permite acompañar a las personas en un camino de desarrollo y mayor bienestar, sobre todo desde las disciplinas de la salud mental. Finalmente, cabe señalar que este artículo no busca ser un examen exhaustivo de análisis entre ambas teorías, sino más bien, un primer paso de aproximación entre dos autores que han presentado una base teórica que expone un camino de aproximación al ser. 


\section{Bibliografía}

Jaspers, K. 1959. Filosofía, II. San Juan de Puerto Rico: Universidad de Puerto Rico.

Keneey, B. 1987. Estética del cambio. Barcelona: Paidós.

Wilber, K. 1984. La conciencia sin fronteras. Aproximaciones de Oriente y Occidente al crecimiento personal. Barcelona: Kairos.

Recibido el 1 Feb 2017

Aceptado el 8 Oct 2017 